\title{
An MCM-Based Microsystem for Colorimetric Detection of Biomolecules in Biological Fluids
}

\author{
Graça Minas, Member, IEEE, Ger de Graaf, Member, IEEE, Reinoud F. Wolffenbuttel, Senior Member, IEEE, \\ and José Higino Correia, Member, IEEE
}

\begin{abstract}
This paper presents a multichip module microsystem for biological fluid analysis. It is composed of three parts, namely 1) CMOS silicon optical detection microsystem, which includes a photodetector and a light-to-frequency converter for readout; 2) dielectric thin-film-based optical filter on top of the photodetector fabricated using IC-compatible postprocessing; and 3) glass die on top containing the microchannels fabricated using SU-8 techniques. The application is in low-cost quantitative measurement of the concentration of biomolecules in biological fluids. Its operation is based on optical absorption in the part of the visible spectrum that is defined by the specific biomolecule. Signals proportional to the intensity of the light transmitted through the biological fluid are available at the output in the form of bit streams, which allows simple computer interfacing. The quantitative measurement of the total protein in urine is successfully demonstrated. The photodiode responsivity is $224 \mathrm{~mA} / \mathrm{W}$ with a full-width at half-maximum of $10 \mathrm{~nm}$ at $\lambda=600 \mathrm{~nm}$. The optical system sensitivity is $1 \mathrm{kHz} / \mathrm{W} \mathrm{m} \mathrm{m}^{-2}$ at $\lambda=670 \mathrm{~nm}$.
\end{abstract}

Index Terms-Biomolecule analysis, multichip module (MCM), optical absorption, $\mathrm{SU}-8$ techniques.

\section{INTRODUCTION}

$\mathbf{F}$ OR diagnostic reasons, patients in a hospital are often subjected to biochemical analysis of their body fluids. Usually, the analyses are carried out in clinical laboratories, and the results become available after several hours, sometimes days. Therefore, a reliable diagnosis cannot be performed within the consultation time. Mistakes in the logistics, such as lost samples and mislabeling, may further delay diagnosis [1]. The automated equipment used in a state-of-the-art laboratory reduces errors, but use of high sample and reagent volumes makes the analysis expensive and does not contribute to patient comfort [2]. Outside the laboratory environment, reagent strips are commercially available [3]. Such strips are intended for a limited set of biomolecules to be analyzed and qualitative color

Manuscript received March 11, 2005; revised September 19, 2005. This work was supported by the Portuguese Foundation of Science and Technology (FCT projects SFRH/BD/1281/2000 and POCTI/3374ESE/1999) and by FEDER. The associate editor coordinating the review of this paper and approving it for publication was Prof. Michael Pishko.

G. Minas and J. H. Correia are with the Department of Industrial Electronics, University of Minho, 4800-058 Guimarães, Portugal (e-mail: gminas@ dei.uminho.pt; higino.correia@dei.uminho.pt).

G. de Graaf and R. F. Wolffenbuttel are with the Department of Microelectronics, Faculty of Electrical Engineering, Mathematics and Computer Science, Delft University of Technology, 2628 CD Delft, The Netherlands (e-mail: g.degraaf@ewi.tudelft.nl; r.f.wolffenbuttel@ewi.tudelft.nl).

Digital Object Identifier 10.1109/JSEN.2006.877992

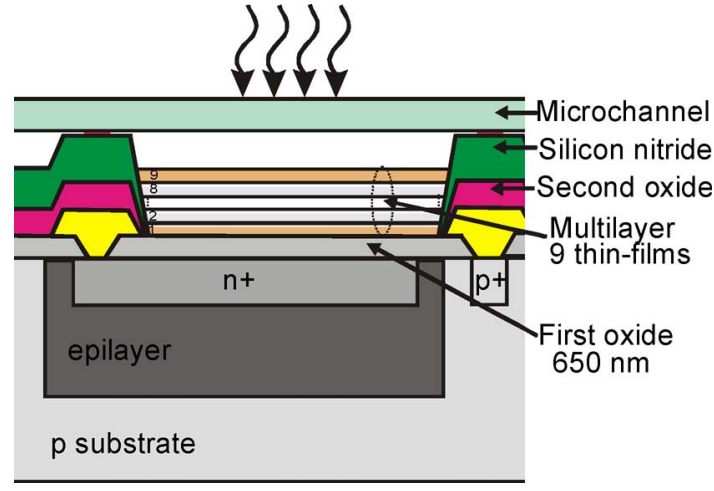

Fig. 1. Cross section of the designed CMOS-compatible optical channel. (Color version available online at http://ieeexplore.ieee.org.)

readout. The need for rapid and on-line measurements with low sample volumes has led to the development of microsystems with the fluidic and detection and readout systems integrated in a single module.

This paper describes a multichip module (MCM)-based microsystem for application in clinical analysis of biological fluids using spectrophotometry. It allows the measurement of the concentration of biomolecules in those fluids. The measurement is based on colorimetric detection by optical absorption in a part of the visible spectrum defined by the reaction of the specific molecule with a specific reagent. The microsystem combines in an MCM the detection and readout system, the optical filtering system, and the microfluidic system.

\section{Microsystem Design}

The optical detection microsystem avoids the need for expensive readout optics and may enable low-cost disposable devices, which would improve the use of spectrophotometric analysis in clinical diagnostics. The optical channel is composed of the fluidic channel, a thin-film optical filter, and a silicon photodetector (see Fig. 1). The structure is optimized for optical response at the absorption peak of a particular biomolecule. The optical absorption intensity is proportional to the biomolecule concentration.

\section{A. Detection and Readout System}

The design involves selection of the photodetector type using the active layers available in a CMOS process and selection 


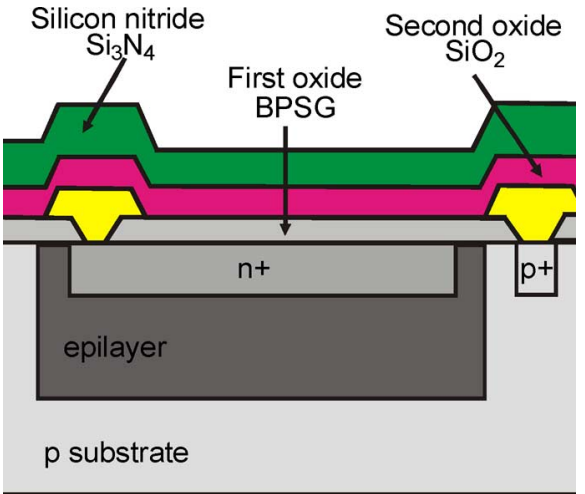

Fig. 2. Cross section of the basic structure of the photodiode for fabrication in a standard $\mathrm{n}$-well CMOS process. (Color version available online at http:// ieeexplore.ieee.org.)

of the dielectric layers for suitable optical response. The photodetector selected is a p-n junction photodiode based on the $\mathrm{n}^{+}$layer/p-epilayer junction. This structure is chosen because it provides the best possible quantum efficiency in the desired spectral range of photodiodes available in a CMOS process [4]. In a standard CMOS process, the junction depth of the photodiodes is fully defined and cannot be altered. The thickness of the $\mathrm{n}^{+}$layer is $350 \mathrm{~nm}$. The thickness of the epilayer is $12 \mu \mathrm{m}$, and the doping concentration is $10^{16} \mathrm{~cm}^{-3}$. However, the quantum efficiency can be improved by a suitable arrangement of dielectric layers on top of the photodiode surface. These act as a thin-film interference filter and influence the optical transmittance for each wavelength independent of the CMOS process. In the CMOS process used, there are three dielectric layers above the photodiode p-n junction (see Fig. 2). The thickness of the first oxide (i.e., boron phosphor silicate glass, BPSG) above the photodiode is measured as $650 \mathrm{~nm}$ and the second oxide $\left(\mathrm{SiO}_{2}\right)$ as $700 \mathrm{~nm}$. The silicon nitride layer (i.e., the overlayer) used for scratch protection is $800 \mathrm{~nm}$ thick. Since technology rules from the standard CMOS process have to be met, the design of the optical path is restricted to combinations of those three dielectric layers. The simulated optical transmissions of those combinations demonstrate that the most suitable photodiode structure for the intended application requires the removal of the second oxide and the silicon nitride layers [5]. This process is performed at the design level, using the same masks designed for the metal contacts and for the overlayer (without additional masks or steps).

A light-to-frequency converter is integrated with the photodiodes to convert the photocurrent into a semidigital signal (see Fig. 3). The reverse-biased junction capacitance of the photodiode $C_{j}$ and capacitor $C_{\mathrm{fb}}$ are used as storage elements. At the voltage $V_{\text {comp }}$ lower than $V_{\text {ref }}$, the comparator output $V_{\text {out }}$ remains at a high logic level. After synchronization with a clock pulse, the analog switch $S_{1}$ is changed for the $A$ position, which forces the capacitor $C_{\mathrm{fb}}$ to be quickly charged during one clock period with the voltage Vdd. Subsequently, this switch is changed again to the $B$ position, and the comparator output voltage commutes to the low logic level $\left(\mathrm{Vdd}>V_{\text {ref }}\right)$. Thus, the photocurrent discharges the capacitor $C_{\mathrm{fb}}$ until the comparator detects $V_{\text {comp }}<V_{\text {ref }}$, which causes $V_{\text {out }}$ to change to the high logic level again and the cycle to repeat (see Fig. 4). The output bit stream frequency is a function of the charge change in the capacitor $\Delta Q$, which is proportional to the input photocurrent [6] and hence to the biomolecule concentration, with $f_{\text {bitstream }}=I_{\text {photodiode }} / \Delta Q$.

Four photodiodes are used in each measurement. The first photodiode is for the reagent (without biomolecules), the second is for the biological fluid being analyzed, the third is for measuring the photodiode dark current, and the fourth is for the calibrator, a standard with a well-known concentration of the biomolecule that is being analyzed. The dark current is the current that flows in a photodiode when there is no optical radiation incident on the photodiode. It is usually measured and subtracted from the flux. As the dark current is temperature dependent, one measurement at the beginning of the experiment is usually not sufficient. Thus, in the reported circuit, a dark current compensation channel is implemented using photodiode 3 . This photodiode is completely covered with metal.

The logic circuit drives the analog switches $S_{2 a}$ through $S_{2 d}$ for selecting the channel to be measured. These switches are identical complementary p-n/MOS switches. The comparator is a clocked high-speed regenerative comparator with a rail-torail input circuit. For reliable operation, a two-phase nonoverlapping clock is used for the analog switch $S_{1}$ and for the comparator. It is possible to use a digital counter for counting the output pulses of the comparator during a fixed time period, producing the digital value correspondent to the photocurrent intensity. Alternatively, a microcontroller can substitute the counter, generating all the control logic and performing additional calculations as well.

\section{B. Optical Filtering System}

An optical filter on top of the photodiode is required with a narrow passband around the wavelength for which the biomolecule being analyzed has its absorption maximum. This enables the selective measurement of the intensity at the desired wavelength transmitted through the mixture. The optical interference filter is based on a nine-layer dielectric thin-film design, which offers high reflectivity with low absorption losses. The multilayer is composed of a stack of $\mathrm{TiO}_{2}$ and $\mathrm{SiO}_{2}$ thin films. The thickness of the films determines the tuned wavelength. $\mathrm{SiO}_{2}$ has been selected because the wavelength dependence of its refractive index for the spectral band between 500 and $700 \mathrm{~nm}$ is almost constant (1.465 and 1.457, respectively). $\mathrm{TiO}_{2}$ has been selected due to fabrication constraints (the deposition process is well characterized).

The multilayer is structurally optimized together with the transmission through the three dielectric layers on top of the p-n junction provided by the CMOS process. The simulations are done with an optics software package TFCalc 3.4, supplied by Software Spectra, Inc., USA. Fig. 5 shows the simulated transmittance of the optical channel (optical effect of photodiode and optical filter) with the layers stack described in Table I. This result shows that the optical channel is sensitive to a single spectral band, with a full-width at half-maximum (FWHM) of $10 \mathrm{~nm}$. The filter can be tuned to cover different spectral 


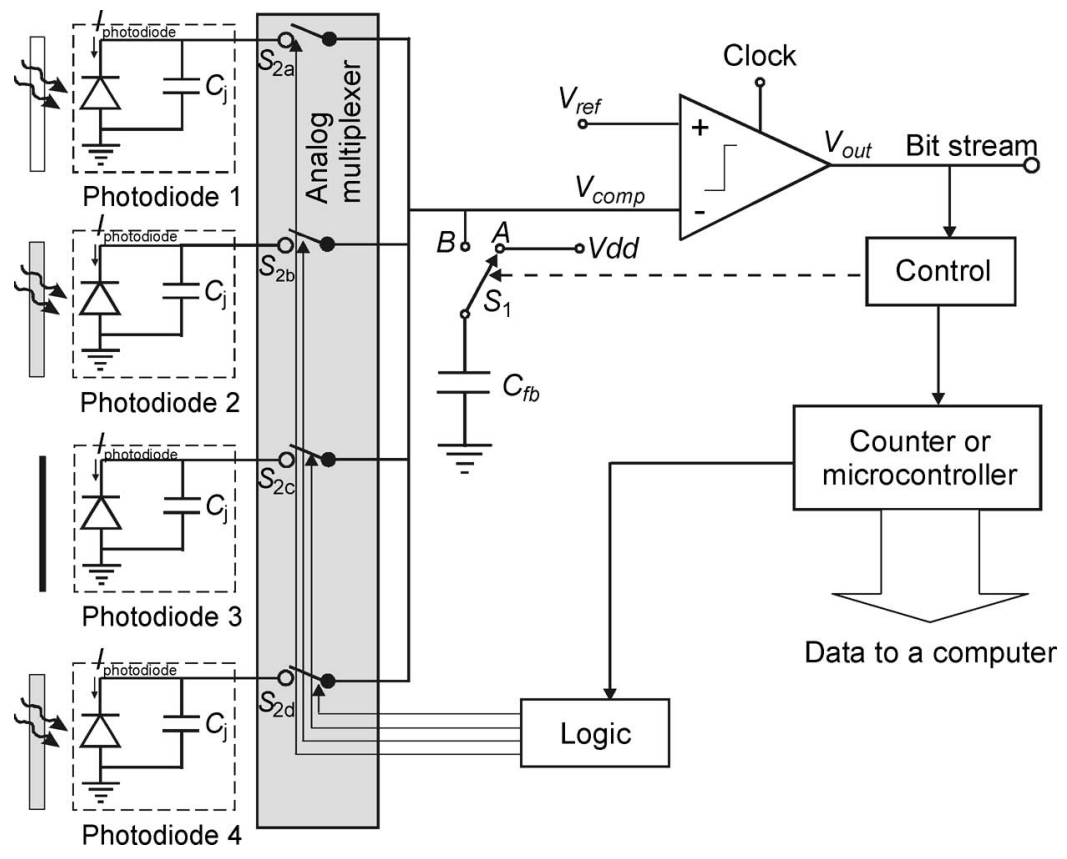

Fig. 3. Block diagram of the photodiode readout circuit.

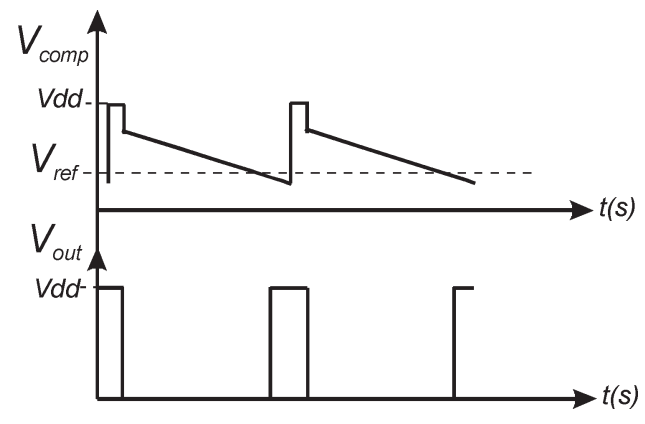

Fig. 4. Comparator input and output voltages.

bands by adjusting the thickness of one or more layers without affecting the microsystem layout.

\section{Microfluidic System}

The microfluidic system contains the microchannels and the detection chambers (see Fig. 6). There are three detection chambers, each $2 \mathrm{~mm}$ wide, $3 \mathrm{~mm}$ long, and $500 \mu \mathrm{m}$ deep. The high depth is crucial for the optical absorption measurements. The detection chamber $\mathbf{A}$ contains only the reagent, and it is needed to obtain the baseline reference and to calibrate the light source. The detection chamber $\mathbf{B}$ allows the mixed solution analysis; it contains the reagent plus the sample with biomolecules. The detection chamber $\mathbf{C}$ is needed to calibrate the biomolecule concentration (with a well-known concentration standard). The main channel is $500 \mu \mathrm{m}$ wide, $70 \mathrm{~mm}$ long, and $500 \mu \mathrm{m}$ deep, with a liquid volume quantity of $20 \mu \mathrm{L}$. It has two inlets, one for the reagent, $\mathbf{R}$, and the other for the sample, $\mathrm{S}$. The chosen structure allows a complete and homogeneous mixing of the reagent with the sample. The mixing process was described in [7].

\section{MiCROSYSTEM FABRICATION}

\section{A. Detection and Readout System}

The CMOS-compatible photodetectors and readout circuits have been fabricated through a double-metal, singlepolysilicon, 1.6- $\mu \mathrm{m} \mathrm{n}$-well CMOS process. The area of each optical channel is $500 \times 500 \mu \mathrm{m}^{2}$. Fig. 7 shows a photograph of the fabricated optical detection microsystem.

\section{B. Optical Filtering System}

The optical filter is postprocessed, by evaporation, on top of the photodetector using the same mask that was used for the selection of the dielectric layers available in the standard CMOS process. The filter fabrication starts with the deposition of an 80-nm $\mathrm{TiO}_{2}$ layer (layer 1 in Table I) after completion of the standard CMOS process, including the metalization and the etching of the two oxide layers on top of the photodiode. Then, the eight subsequent layers of $\mathrm{SiO}_{2}$ and $\mathrm{TiO}_{2}$ are deposited with the thicknesses described in Table I. A scanning electron microscopy (SEM) photograph of the cross section of the optical channel is shown in Fig. 8. A commercially available passband optical filter on top of the MCM-based microsystem is used to block the nonvisible part of the spectrum.

\section{Microfluidic System}

Fig. 9 shows a photograph of the microchannels fabricated using SU-8 techniques. The SU-8-based fabrication is a lowcost process, biocompatible, UV lithography semiconductor compatible, and does not require expensive masks. Moreover, SU-8-based processing enables the fabrication of deep microchannels with very low sidewall roughness and is suitable for optical absorption measurement [8]. 


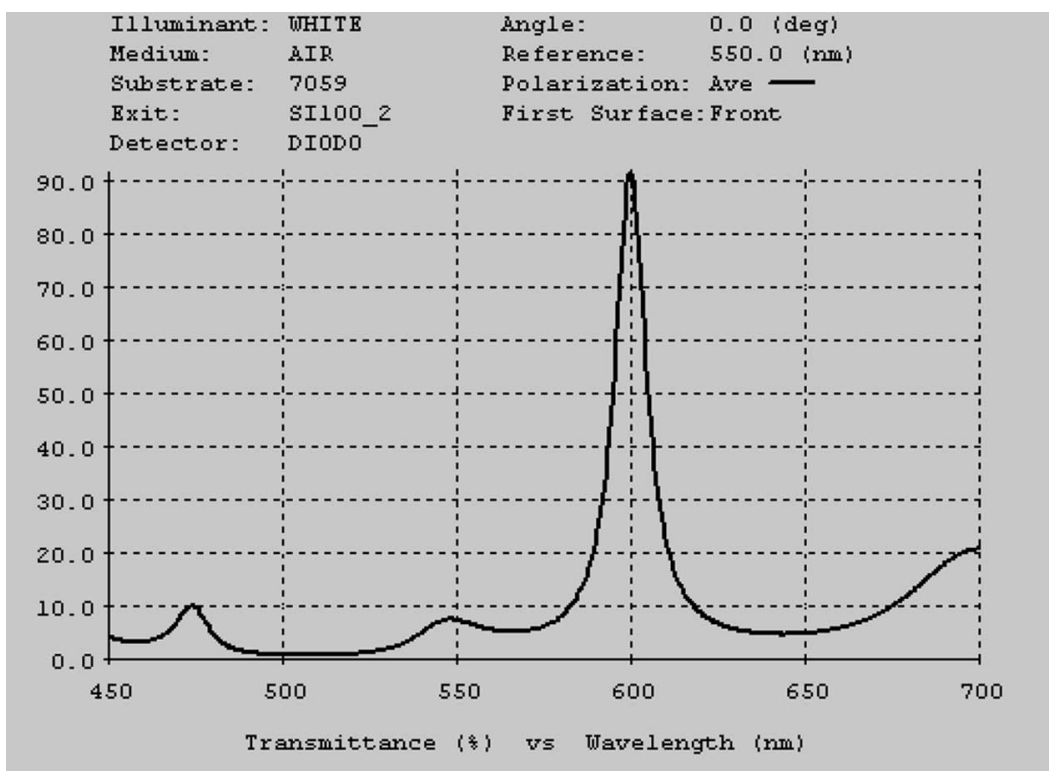

Fig. 5. Simulated spectral transmittance of the optical filtering channel.

TABLE I

LAYERS MATERIAL AND THICKNESSES OF THE OPTICAL FILTERING CHANNEL

\begin{tabular}{lll}
\hline \hline \multicolumn{1}{c}{ Layer } & Material & \multicolumn{1}{c}{ Thickness } \\
\hline 9 & $\mathrm{TiO}_{2}$ & $45 \mathrm{~nm}$ \\
8 & $\mathrm{SiO}_{2}$ & $65 \mathrm{~nm}$ \\
7 & $\mathrm{TiO}_{2}$ & $60 \mathrm{~nm}$ \\
6 & $\mathrm{SiO}_{2}$ & $90 \mathrm{~nm}$ \\
5 & $\mathrm{TiO}_{2}$ & $120 \mathrm{~nm}$ \\
4 & $\mathrm{SiO}_{2}$ & $60 \mathrm{~nm}$ \\
3 & $\mathrm{TiO}_{2}$ & $45 \mathrm{~nm}$ \\
2 & $\mathrm{SiO}_{2}$ & $70 \mathrm{~nm}$ \\
1 & $\mathrm{TiO}_{2}$ & $80 \mathrm{~nm}$ \\
$1^{\text {st }}$ oxide & $\mathrm{SiO}_{2}$ & $650 \mathrm{~nm}$ \\
Diode & $\mathrm{Si}^{\text {Ex }}$ & Exit medium \\
\hline \hline
\end{tabular}

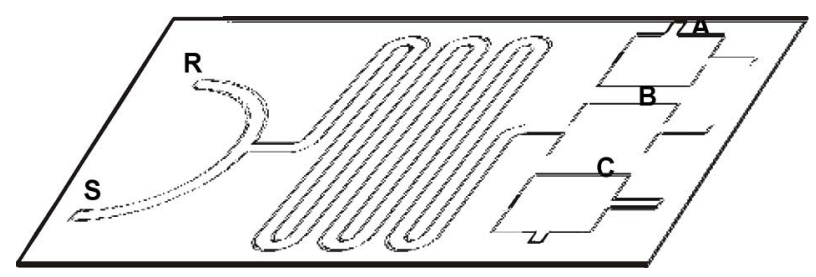

Fig. 6. Layout of the microfluidic system.

A negative mask of the microchannels' die layout is fabricated from a regular transparency foil (like the one used in printed circuit boards). The SU-8 photoresist chosen is the SU-8 100. The microchannel fabrication starts with spinning of the SU-8 photoresist on a glass substrate at $700 \mathrm{r} / \mathrm{min}$ for $100 \mathrm{~s}$. Soft bake prior to exposure is performed at $90{ }^{\circ} \mathrm{C}$ for $3000 \mathrm{~s}$. The second spun is carried out at $700 \mathrm{r} / \mathrm{min}$ for $100 \mathrm{~s}$, and a soft bake for $300 \mathrm{~s}$ at $50{ }^{\circ} \mathrm{C}$ is again performed. The temperature is ramped up to $90{ }^{\circ} \mathrm{C}$ for $5400 \mathrm{~s}$ and subsequently ramped down again. The exposure takes $900 \mathrm{~s}$ on an Electronic Vision EV-420 mask and bonding aligner. Postexposure bake is performed for

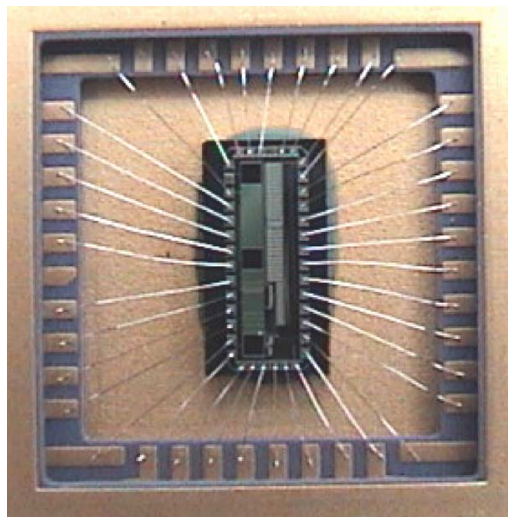

Fig. 7. Photograph of the optical detection microsystem. (Color version available online at http://ieeexplore.ieee.org.)

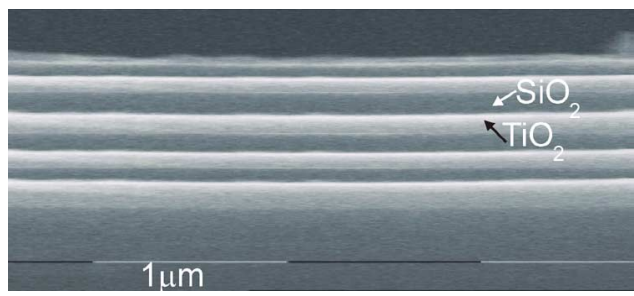

Fig. 8. SEM photograph showing the cross section of the optical filter. (Color version available online at http://ieeexplore.ieee.org.)

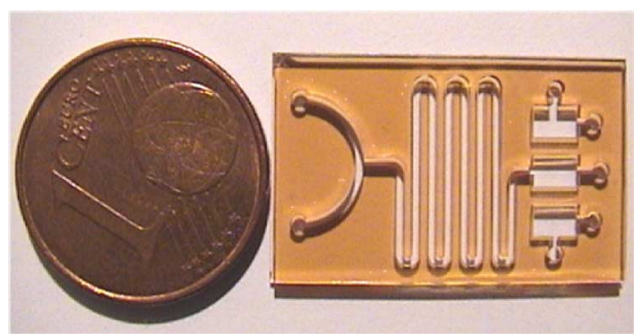

Fig. 9. Photograph of the SU-8-based structure microchannels. (Color version available online at http://ieeexplore.ieee.org.) 


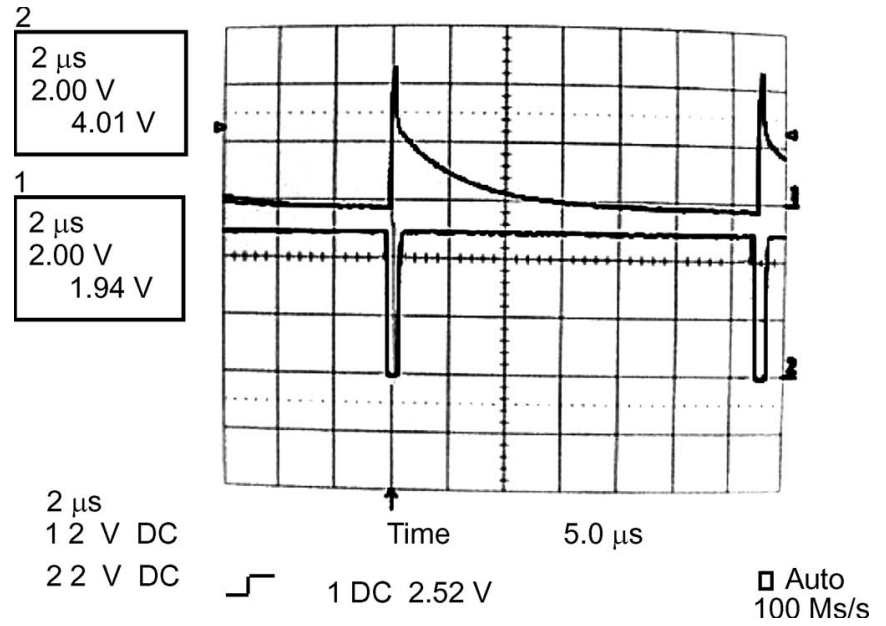

Fig. 10. Oscilloscope capacitor voltage (1) and inverted comparator output voltage (2) for a photodiode current of $137.0 \mathrm{nA}$.

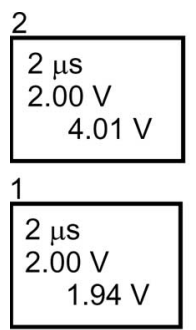

$2 \mu \mathrm{s}$

$12 \mathrm{~V}$ DC

$22 \mathrm{VDC}$

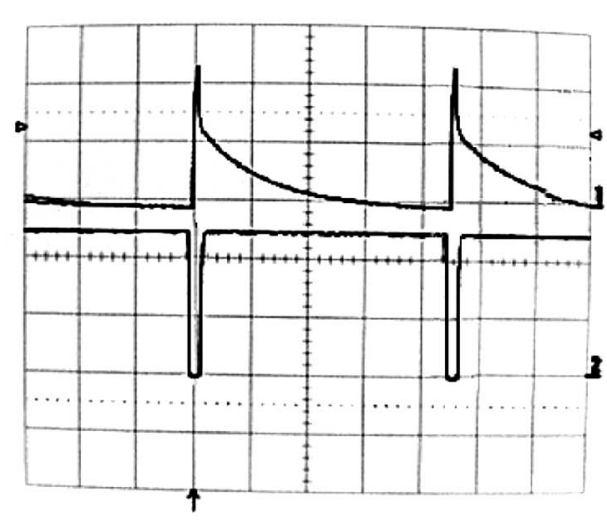

Time $\quad 5.0 \mu \mathrm{s}$

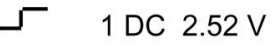

Auto

$100 \mathrm{Ms} / \mathrm{s}$
Fig. 11. Oscilloscope capacitor voltage (1) and inverted comparator output voltage (2) for a photodiode current of $138.1 \mathrm{nA}$.

$300 \mathrm{~s}$ at $50{ }^{\circ} \mathrm{C}$. The temperature is again ramped up to $90{ }^{\circ} \mathrm{C}$ for $1200 \mathrm{~s}$ and ramped down. The fabrication ends with the development during $2700 \mathrm{~s}$ in the SU-8 developer.

\section{EXPERIMENTAL RESUlTS}

The electrical characteristics and spectral responsivity were measured using a Keithley 487 picoamperemeter (full-scale range from $10 \mathrm{fA}$ to $2 \mathrm{~mA}$ and a resolution of $51 / 2$ digit), and a $250-\mathrm{W}$ quartz tungsten halogen lamp with the ORIEL Cornerstone 130 monochromator was used as light source. The measurements were calibrated with a calibrated commercially available photodiode as reference (Hamamatsu S1336-5BQ).

The oscilloscope traces in Figs. 10 and 11 show the electrical characteristics of the readout circuit. These signals were obtained for a photodiode current of 137.0 and $138.1 \mathrm{nA}$, which represents 15 and $1 \mathrm{mg} / \mathrm{dl}$ of the total protein concentration in urine, respectively. The output frequency of the light-tofrequency converter for different values of the photodiode current is shown in Fig. 12. The photodiode dark current is

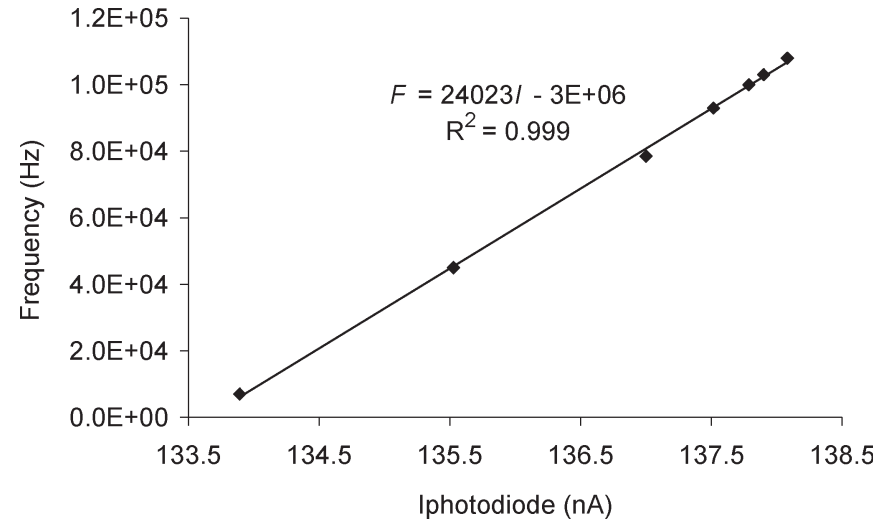

Fig. 12. Output frequency of the light-to-frequency converter for different photodiode currents ( $F$, frequency; $I$, photodiode current; $R$, Pearson product moment correlation coefficient).

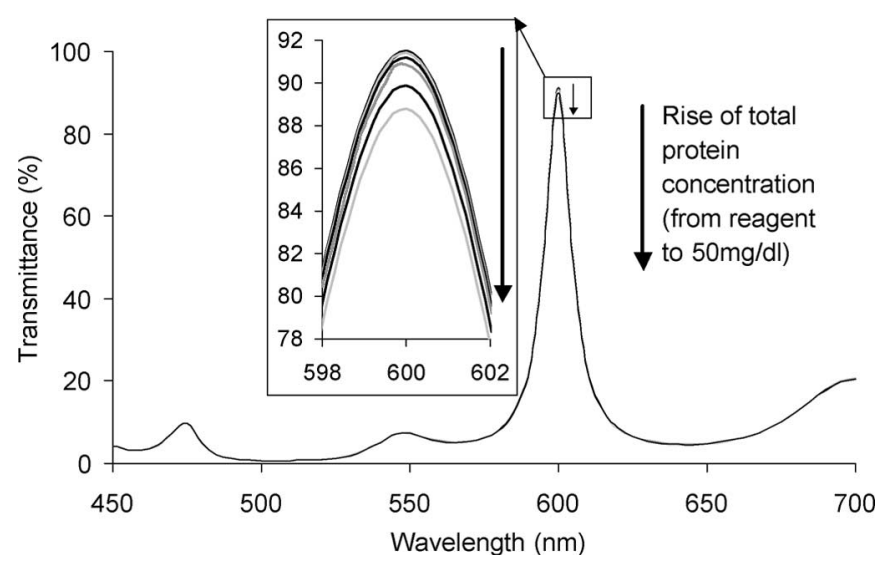

Fig. 13. Measured transmittance spectra for different total protein concentrations (optical effect of photodiode and optical filter included).

$0.38 \mathrm{pA}\left(1.52 \times 10^{-18} \mathrm{~A} / \mu \mathrm{m}^{2}\right)$ at $0 \mathrm{~V}$, its responsivity is $224 \mathrm{~mA} / \mathrm{W}$ at $\lambda=600 \mathrm{~nm}$ (the wavelength at which the total protein in urine has its absorption maximum), and the sensitivity achieved is $1 \mathrm{kHz} / \mathrm{W} \mathrm{m}{ }^{-2}$ at $\lambda=670 \mathrm{~nm}$ (using the TLS230 from Texas Instruments as a reference).

The total protein concentration in a urine sample was measured as a test of this MCM-based microsystem. The reagent used in the measurements was the Microprotein-PR reagent from Sigma-Aldrich. It reacts with a sample of urine containing total protein in a 50:1 ratio and produces an absorption maximum at a specific wavelength $(\lambda=600 \mathrm{~nm})$.

Fig. 13 shows the measured transmittance through the entire optical channel as a function of protein concentration. Measurements were performed in a range between 1 and $50 \mathrm{mg} / \mathrm{dl}$, which includes normal and abnormal values in a human being (normal values are typically less than $20 \mathrm{mg} / \mathrm{dl}$ ). The transmittance is defined as $T=I / I_{0}$, where $I$ is the measured photodiode current for each mixture, and $I_{0}$ is the measured photodiode current of the reagent. The difference in the transmittance value for different concentrations is small once the total protein appears in small quantities in human urine despite its composition, which includes hundred of proteins [2]. The reproducibility variation coefficients for ten different measurements and ten different assays are less than $10 \%$. The 


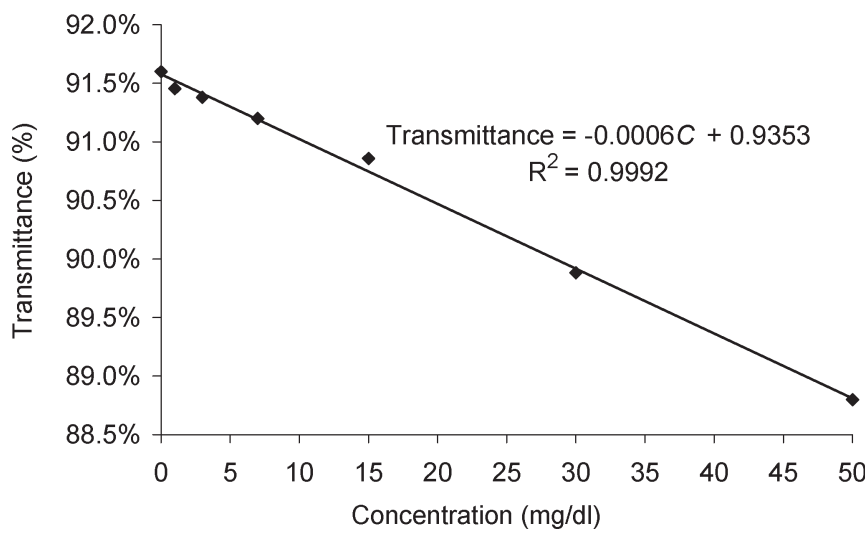

Fig. 14. Calibration curve for different total protein concentrations at $\lambda=$ $600 \mathrm{~nm}(C$, concentration value; $R$, Pearson product moment correlation coefficient).

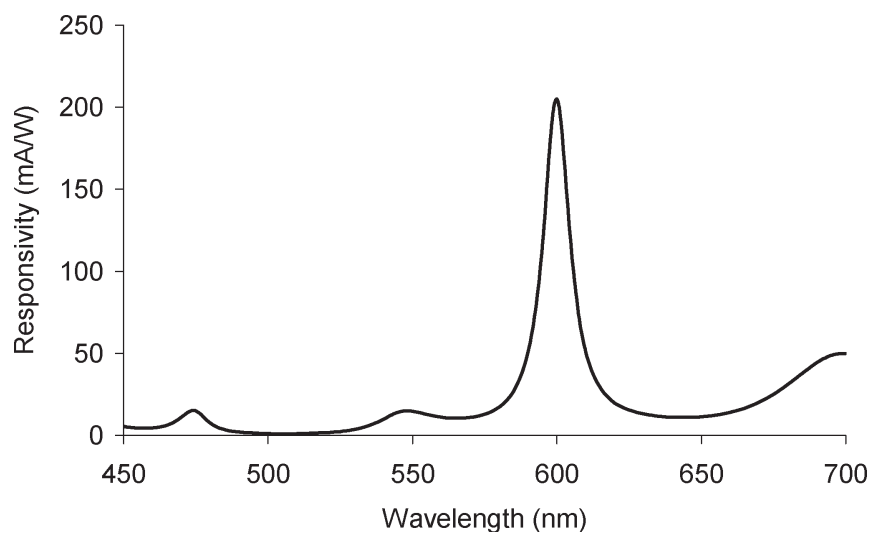

Fig. 15. Measured spectral responsivity without protein biomolecules (optical effect of photodiode and optical filter included).

transmittance at $\lambda=600 \mathrm{~nm}$ as a function of the different total protein concentrations is shown in Fig. 14.

Fig. 15 presents the spectral responsivity for the optical channel (optical effect of the photodiode and the optical filter) measured without the total protein concentration (only with the reagent). The presence of the optical filter limits the light transmitted to the underlying photodiode. However, once it is composed of high-performance dielectric layers, the light transmitted through the optical filter is $92 \%$ of the incident light.

The output frequency of the light-to-frequency converter when the MCM-based microsystem is tested for measuring the different total protein concentrations in urine is presented in Fig. 16. This frequency is proportional to the input photocurrent (see Fig. 12) and, hence, to the total protein concentration.

The measurements confirm the direct proportional dependence between the intensity of the color produced by the mixture and the total protein concentration. The calibration curve of Fig. 14 shows a linear behavior at the peak of the absorption spectrum at $600 \mathrm{~nm}$. These results agree with macroscopic measurements performed with well-known total protein standards and using state-of-the-art laboratory equipment [9]. It can also be concluded that the fabricated optical channel is sensitive to a narrow spectral band centered at the wavelength for which the biomolecule being analyzed has its absorption maximum and with a FWHM $=10 \mathrm{~nm}$.

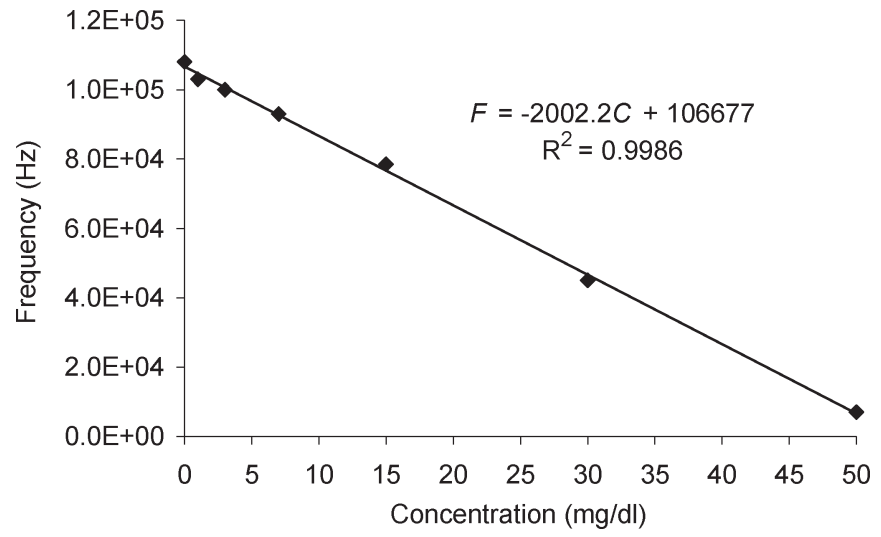

Fig. 16. Output frequency of the MCM-based microsystem for different total protein concentrations ( $F$, frequency; $C$, concentration value; $R$, Pearson product moment correlation coefficient).

\section{CONCLUSION}

The MCM microsystem presented here offers a new approach for clinical analysis, especially in biological fluid analysis, due to its portability, ensuring that the analysis can be performed at any location with instantaneous results. Moreover, its optical detection and filtering system avoids the need for expensive readout optics and opens the door to low-cost disposable devices. The performance was successfully demonstrated in the quantitative measurement of the total protein in urine. The photodiode responsivity is $224 \mathrm{~mA} / \mathrm{W}$ with a FWHM of $10 \mathrm{~nm}$ at the wavelength at which the total protein concentration has its absorption maximum, i.e., at $\lambda=600 \mathrm{~nm}$. The photodiode sensitivity was $1 \mathrm{kHz} / \mathrm{W} \mathrm{m}^{-2}$ at $\lambda=670 \mathrm{~nm}$, using the TLS230 from Texas Instruments as reference.

\section{ACKNOWLEDGMENT}

The authors would like to thank P. Turmezei from the Laboratory for Electronic Instrumentation, TUDelft, The Netherlands, for his help with the SU-8 processing. This work was sponsored by FCT/REEQ/379/EEI/2005.

\section{REFERENCES}

[1] P. Connolly, "Clinical diagnostics opportunities for biosensors and bioelectronics," Biosensors and Bioelectron., vol. 10, no. 1/2, pp. 1-6, 1995

[2] S. K. Strasinger and M. S. Di Lorenzo, Urianalysis and Body Fluids, 4th ed. Philadelphia, PA: Davis, 2001.

[3] [Online]. Available: http://www.hypoguard.com/diascreen_reagent_strips. html

[4] B. Fowler, A. El Gamal, and D. X. D. Yang, "A CMOS area image sensor with pixel-level A/D conversion," presented at the ISSCC Conf., San Francisco, CA, Feb. 1994, TP 13.5.

[5] G. Minas, J. S. Martins, J. C. Ribeiro, R. F. Wolffenbuttel, and J. H. Correia, "Biological microsystem for measuring uric acid in biological fluids," Sens. Actuators A, Phys., vol. 110, no. 1-3, pp. 33-38, Feb. 2004.

[6] G. de Graaf and R. F. Wolffenbuttel, "Optical CMOS sensor system for detection of light sources," Sens. Actuators A, Phys., vol. 110, no. 1-3, pp. 77-81, Feb. 2004.

[7] G. Minas, J. C. Ribeiro, R. F. Wolffenbuttel, and J. H. Correia, "2-D modeling and simulation of fluidic microsystems for biological fluids analysis," in Proc. MME, Delft, The Netherlands, Nov. 2-4, 2003, pp. 239-485.

[8] J. D. Gelorme, R. J. Cox, and S. A. R. Gutierrez, "Photoresist composition and printed circuit boards and packages made therewith (IBM)," U.S. Patent 4882 245, 1989.

[9] N. Watanabe et al., "Urinary protein as measured with a pyrogallol red molybdate complex, manually and in a Hitachi 726 automated analyzer," Clin. Chem., vol. 32, no. 8, pp. 1551-1554, Aug. 1986. 


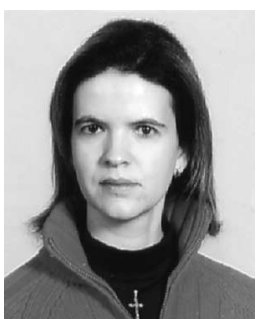

Graça Minas (S'96-M'05) received the B.Sc., M.Sc., and Ph.D. degrees from the University of Minho, Guimarães, Portugal, in 1994, 1998, and 2004, respectively, all in industrial electronics engineering. Her thesis work was in cooperation with the Laboratory for Electronic Instrumentation, Delft University of Technology, Delft, The Netherlands, and dealt with lab-on-a-chips for biological fluids analysis.

Between 1995 and 2004, she was a Lecturer and, since 2004, she has been an Auxiliary Professor with the Department of Industrial Electronics, University of Minho, where she is involved in biomedical microdevices research.

Prof. Minas won the second place for the Best Oral Presentation in the Eurosensors XVII.

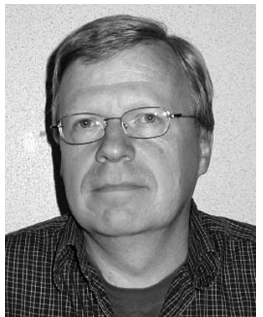

Ger de Graaf (M'98) received the B.S.E.E. degree in electrical and control engineering from the Technische Hogeschool, Rotterdam, The Netherlands, in 1983.

Since 1976, he has been a Staff Member with the Department for Microelectronics, Faculty for Information Technology and Systems, Delft University of Technology, Delft, The Netherlands. Currently, he is working on electronic circuits for silicon sensors. In addition, since 1992, he has a consultancy company specializing in electronic design and computercontrolled measurement systems.

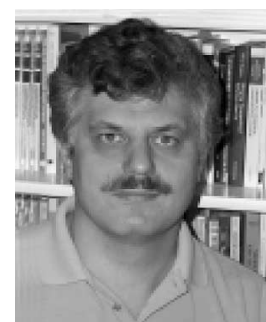

Reinoud F. Wolffenbuttel (S'86-M'88-SM'97) received the M.Sc. and Ph.D. degrees from Delft University of Technology, Delft, The Netherlands, in 1984 and 1988, respectively.

Between 1986 and 1993, he was an Assistant Professor and, since 1993, he has been an Associate Professor with the Department of Microelectronics, Faculty of Information Technology and Systems, Delft University of Technology, where he is involved in instrumentation and measurement, in general, and on-chip functional integration of microelectronic circuits and silicon sensor, IC process compatible MEMS fabrication, and silicon microsystems, in particular. He was a Visitor at the University of Michigan, Ann Arbor, in 1992, 1999, and 2001, at Tohoku University, Sendai, Japan, in 1995, and at EPFL, Lausanne, Switzerland, in 1997.

Prof. Wolffenbuttel served as the General Chairman of the Dutch National Sensor Conference in 1996, the Eurosensors in 1999, and the MicroMechanics Europe Workshop in 2003. He was a recipient of the 1997 NWO Pioneer Award.

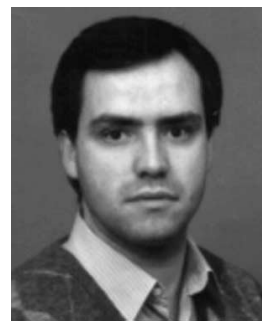

José Higino Correia (S'96-M'00) received the B.S degree in physical engineering from the University of Coimbra, Coimbra, Portugal, in 1990 and the Ph.D. degree from the Laboratory for Electronic Instrumentation, Delft University of Technology, Delft, The Netherlands, in 1999. His Ph.D. dissertation was in the field of microsystems for optical spectral analysis.

He is currently an Associate Professor with the Department of Industrial Electronics, University of Minho, Guimarães, Portugal. His professional interests are in micromachining and microfabrication technology for mixed-mode systems, solid-state integrated sensors, microactuators, and microsystems.

Prof. Correia was the General Chairman of the Eurosensors 2003, Guimarães. 